\title{
Development of ichthyosiform skin compensates for defective permeability barrier function in mice lacking transglutaminase 1
}

\author{
Nobuo Kuramoto, ${ }^{1}$ Toshihiro Takizawa, ${ }^{2,3}$ Takami Takizawa, ${ }^{2,3,4}$ Masato Matsuki, ${ }^{1}$ \\ Hiroyuki Morioka, ${ }^{5}$ John M. Robinson, ${ }^{3}$ and Kiyofumi Yamanishi ${ }^{1}$
}

\author{
${ }^{1}$ Department of Dermatology, Kyoto Prefectural University of Medicine, Kyoto, Japan \\ ${ }^{2}$ Department of Anatomy, Jichi Medical School, Tochigi-ken, Japan \\ ${ }^{3}$ Department of Physiology and Cell Biology, Ohio State University, Columbus, Ohio, USA \\ ${ }^{4}$ Cosmetics Laboratory, Kanebo Ltd., Kanagawa-ken, Japan \\ ${ }^{5}$ Division of Electron Microscopy, Central Laboratories, Kyoto Prefectural University of Medicine, Kyoto, Japan \\ Address correspondence to: Kiyofumi Yamanishi, Department of Dermatology, \\ Kyoto Prefectural University of Medicine, Kamigyo-ku, Kyoto 602-8566, Japan. \\ Phone: 81-75-251-5587; Fax: 81-75-251-5384; E-mail: kyamanis@koto.kpu-m.ac.jp.
}

Received for publication June 19, 2001, and accepted in revised form November 15, 2001.

Transglutaminase 1 (TGase 1) is one of the genes implicated in autosomal recessive congenital ichthyosis. Skin from TGase $1^{-/-}$mice, which die as neonates, lacks the normal insoluble cornified envelope and has impaired barrier function. Characterization of in situ dye permeability and transepidermal water loss revealed defects in the development of the skin permeability barrier in TGase $1^{-/-}$mice. In the stratum corneum of the skin, tongue, and forestomach, intercellular lipid lamellae were disorganized, and the corneocyte lipid envelope and cornified envelope were lacking. Neonatal TGase $1^{-/}$mouse skin was taut and erythrodermic, but transplanted TGase $1^{-/}$mouse skin resembled that seen in severe ichthyosis, with epidermal hyperplasia and marked hyperkeratosis. Abnormalities in those barrier structures remained, but transepidermal water loss was improved to control levels in the ichthyosiform skin. From these results, we conclude that TGase 1 is essential to the assembly and organization of the barrier structures in stratified squamous epithelia. We suggest that the ichthyosiform skin phenotype in TGase 1 deficiency develops the massive hyperkeratosis as a physical compensation for the defective cutaneous permeability barrier required for survival in a terrestrial environment.

J. Clin. Invest. 109:243-250 (2002). DOI:10.1172/JCI200213563.

\section{Introduction}

Autosomal recessive congenital ichthyosis (ARCI) is the clinical entity of keratinization disorders characterized by generalized ichthyosiform skin phenotypes. These have been classically subdivided into at least two major subtypes, lamellar ichthyosis (LI) (MIM 242300) (1) and nonbullous congenital ichthyosiform erythroderma (CIE) (MIM 242100) $(2,3)$, although ARCI consists of more heterogeneous disorders with a variety of distinct features. Patients with ARCI are often born as "collodion babies," encased in translucent, collodion-like membranes (4). This condition is sometimes life-threatening with severe dehydration due to high transepidermal water loss (TEWL) (5). In LI patients that survive, the membrane sheds within a few weeks and the skin phenotype changes into an ichthyosiform appearance. As they grow, LI patients become covered with large, brownish, platelike desquamations (1), which are accompanied by ectropions and occasional alopecia (6). In contrast, in $\mathrm{CIE}$, scales are fine and thinner and erythroderma is more prominent. Linkage studies and mutation analyses have revealed that $\mathrm{LI}$ is associated with mutations of the transglutaminase 1 (TGase 1) gene on 14q11.2 $(7,8)$, and at the 2q33-q35 (9) and 19p12-q12 (10) loci, whereas nonbul- lous CIE is associated with TGase $1(6,11)$, and at the $3 \mathrm{p} 21$ locus (10). In addition, another ARCI locus for one Finnish family with a nonlamellar, nonerythrodermic phenotype has been assigned to 19p13.1-p13.2 (12).

$\mathrm{ARCI}$ is also classified into four ichthyosis congenita (IC) groups, based on the ultrastructure of the stratum corneum (SC) (13). Of these, the clinical symptoms of IC types I and II roughly correspond to CIE and LI, respectively $(14,15)$. TGase 1 mutations have been detected in both IC type I and type II, but more frequently in IC type II (16). TGase 1 (keratinocyte TGase) is a membrane-bound isoform of the TGase family (E.C. 2.3 .2 .13$)$ that catalyzes $\varepsilon$ - $(\gamma$-glutamyl) lysine crosslinking reactions and that participates in a wide variety of biological processes (reviewed in ref. 17). In the SC of stratified squamous epithelia, the cell periphery is lined with a cornified envelope (CE), an approximately 15-nm-thick, membranous structure composed of $\varepsilon$-( $\gamma$-glutamyl) lysine cross-linked proteins including involucrin, loricrin, and various other peptides (reviewed in ref. 18). These tightly cross-linked proteins possibly confer on the SC its mechanical strength and its resistance against chemical and microbial invasions. TGase 1 is able to produce high-molecular weight poly- 
mers by cross-linking those substrate proteins, whereas another isozyme, TGase 3, is only able to oligomerize those proteins (19). TGase 1 knockout (TGase $1^{-/}$) mice lack the CE, which indicates that TGase 1 is essential for formation of the CE in vivo (20). The ultrastructural features of IC type I include numerous intracellular lipid droplets within corneocytes. IC type II has a characteristic thin CE as well as lipid droplets and/or crystalloid inclusions (cholesterol crystals) within the cells. TGase 1 catalytic activity and its protein levels are actually decreased in cultured keratinocytes derived from LI patients with TGase 1 mutations $(8,21)$. Regardless of the progress in these biological and genetic analyses of TGase 1, the mechanism(s) by which TGase 1 mutations lead to the ichthyosiform skin phenotypes of LI and CIE has not been delineated.

TGase $1^{-/}$mice die soon after birth with impaired skin barrier functions (20). The skin permeability barrier is maintained by lipid lamellar bilayers organized in the intercellular matrix of the SC (reviewed in ref. 22). The intercellular lipids include ceramides, cholesterol, and fatty acids, together with smaller amounts of phospholipids and glucosylceramides (reviewed in ref. 23). The lipid lamellae are formed by the rearrangement of lamellar disks released from lamellar granules into the interface of the SC and the uppermost cells of the granular layer (24). The minimum unit of the lipid membrane is the double bilayer, whose layers interact with each other and also with the adjacent corneocyte lipid envelope (CLE) (25). The CLE is an approximately 5 -nm-thick lipid monolayer that is covalently attached to the surface of the $\mathrm{CE}(26,27)$. $\omega$-Hydroxyceramide, a major component of the CLE, is linked by an ester bond to involucrin and other constituent peptides of the $\mathrm{CE}(26,28,29)$, also probably via TGase 1 enzyme activity (30). The CLE is believed to function as a scaffold for the deposition of intercellular lamellae to maintain permeability barrier homeostasis (31). In some cases of LI and CIE, incompletely formed intercellular lamellae and disorganized lamellar arrays have been found (32). However, it has not been documented whether those abnormalities in the SC interstices are associated with TGase 1 mutations.

In this study, we demonstrate that TGase 1 is essential for the development of the skin permeability barrier and for the organization of the SC intercellular lipid lamellar structure of stratified squamous epithelia, possibly via formation of the CLE. Moreover, we show evidence that skin lacking TGase 1 develops an ichthyosiform phenotype as it matures, which suggests that this particular phenotype may be due to a physical compensation for the impaired skin barrier by massive hyperkeratosis.

\section{Methods}

Animals. All studies involving animals were reviewed and approved by the Animal Use and Care Committee of the Kyoto Prefectural University of Medicine. All animals used for this study were maintained under pathogenfree conditions. TGase $1^{-/-}$mice were generated by inter- crossing of TGase $1^{+/-}$mice $(129 / \mathrm{Sv} \times \mathrm{C} 57 \mathrm{BL} / 6)$, as described previously (20). TGase $1^{+/-}$mice were timemated within 2 hours and the midpoint of the mating time was designated as gestational age 0 (33). Days postcoitum (dpc) were calculated from the time designated as 0 . Genotypes of embryos and neonates were determined by PCR analysis of tail DNA using three primers, P3 (5'-CCAAAGGCCTACCCGCTTCCATTGCTCAGC-3'), P4 (5'- AGCTACTGGGGGCGGGCGGGTGACTCTTCTA-3'), and P5 (5'-GCGTAGGTTTAGGTTGTGTCCGTTGTTCTTAG- $3^{\prime}$ ). Primers $\mathrm{P} 3$ and $\mathrm{P} 5$ were designed to distinguish the $0.7-\mathrm{kb}$ targeted allele band from the $0.5-\mathrm{kb}$ wild-type band amplified by primers P4 and P5. As controls for these experiments, we used TGase $1^{+/-}$or wild-type littermates of TGase $1^{-/-}$mice.

Skin grafting. Five control and six TGase $1^{-/-}$neonates were killed by decapitation, and their dorsal skins were excised and transplanted onto athymic nude mice. Those grafted skins were fully adapted within 1 week of grafting. Two weeks after the transplantation, the permeability barrier function of the grafted skins was assessed, and those skins were then harvested for histological analysis.

Measurement of TEWL. TEWL from skins of embryonic and neonatal mice and from skins grafted on nude mice was examined under normal conditions, using a Courage and Khazaka Tewameter TM210 (Courage and Khazaka Electronic Co., Cologne, Germany), as described previously (20). Data are expressed in $\mathrm{g} / \mathrm{m}^{2} / \mathrm{h}$ as means \pm SD of nine different measurements. The statistical significance of data was estimated using a one- or two-way ANOVA repeated measure with Scheffe's test for grafted skins or for embryonic and neonatal mice, respectively.

Skin permeability assay. The in situ skin permeability assay using toluidine blue was performed as described by Hardman et al. (33). Embryos and neonatal mice were rinsed in PBS and immersed in 25\%,50\%,75\%, and $100 \%$ methanol for 1 minute each successively; they were then rehydrated in PBS and stained in $0.1 \%$ toluidine blue for 10 minutes at room temperature. They were briefly washed in PBS and were immediately photographed.

Preparation of CEs. Excised dorsal skins of 18.5-dpc mice were immersed in PBS containing 10 mM EDTA at room temperature for 1 hour to separate the epidermis. Epidermal sheets were heated in 2\% SDS extraction buffer (0.1 mM Tris [pH 8.5] and $20 \mathrm{mM}$ DTT, $5 \mathrm{mM}$ EDTA, $2 \% \mathrm{SDS}$ ) at $95^{\circ} \mathrm{C}$ for 10 minutes (34). Insoluble CEs were precipitated by centrifugation at $9,000 \mathrm{~g}$ for 15 minutes.

Immunobistochemistry and immunofluorescence. Tissues were fixed in $10 \%$ formaldehyde in PBS, then embedded in paraffin. Those tissues were sectioned at $4 \mu \mathrm{m}$ thickness, and deparaffinized sections were subjected to immunohistochemistry as well as to hematoxylin-andeosin staining. A Vectorstain Universal kit (Vector Laboratories Inc., Burlingame, California, USA) was used for immunohistochemistry according to the manufacturer's instructions. The rabbit polyclonal anti-mouse pro- 

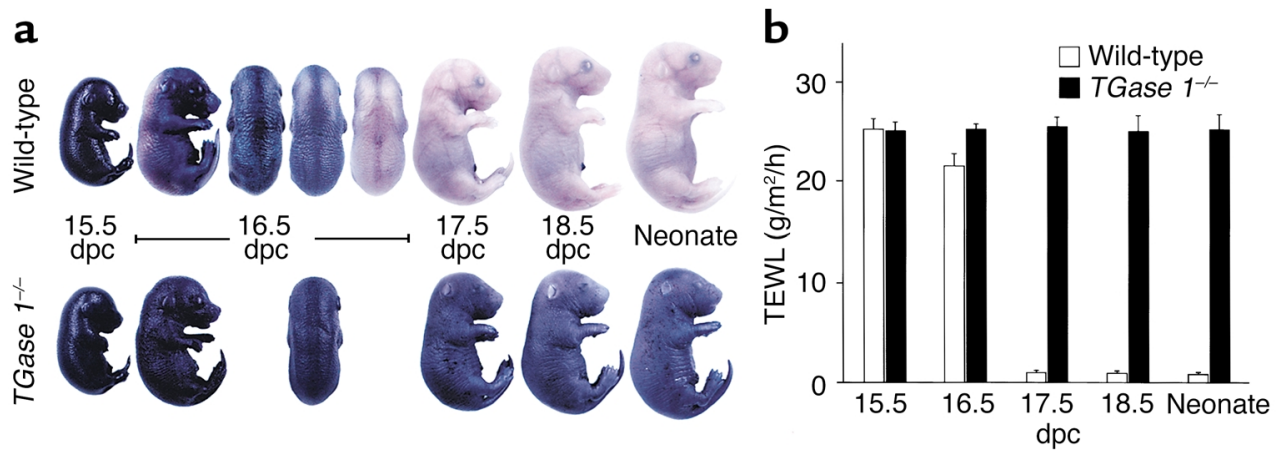

c

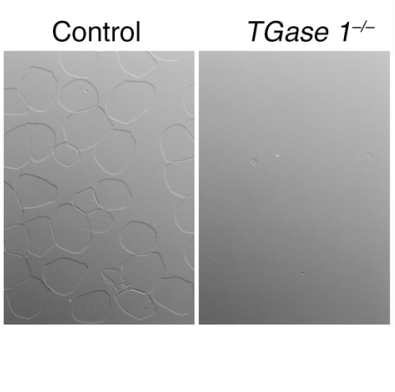

\section{Figure 1}

Defective development of the skin permeability barrier and loss of insoluble CEs in TGase $1^{-/-}$mice. (a) In situ dye permeability. The entire skin of the wild-type embryo at $15.5 \mathrm{dpc}$ is permeable to toluidine blue. Wild-type embryos at $16.5 \mathrm{dpc}$ become partially impermeable to the dye in a variable patterning manner dorsolaterally, and at $17.5 \mathrm{dpc}$ and thereafter their skin is resistant to the stain. In contrast, TGase $1^{-/-}$embryos and neonates are totally permeable to the dye at all times during their development. (b) Time course of TEWL in late embryogenesis. In wildtype embryos at $15.5 \mathrm{dpc}$, TEWL is high, but at $16.5 \mathrm{dpc}$, the level of TEWL begins to decrease $(P<0.0001)$, and at $17.5 \mathrm{dpc}$ and later, TEWL is decreased to the level of neonates. In contrast, the TEWL levels in TGase $1^{-/-}$embryos and neonates remain as high as at $15.5 \mathrm{dpc}$. Error bars represent SD of nine different measurements at each time point. (c) Nomarski contrast image of CEs. Intact CEs are isolated in abundance from the epidermis of wild-type neonates (left). However, CEs are almost completely absent in the TGase $1^{-/-}$epidermis (right).

liferating cell nuclear antigen (PCNA) antibody used as a primary antibody was purchased from Sigma Chemical Co. (St. Louis, Missouri, USA). Immunofluorescence for involucrin and loricrin was performed as described previously using rabbit polyclonal mouse loricrin and mouse involucrin antibodies (Covance Research Products Inc., Richmond, California, USA) (35).

Electron microscopy. Freshly obtained samples were fixed in $2 \%$ paraformaldehyde and $2.5 \%$ glutaraldehyde in $0.1 \mathrm{M}$ sodium cacodylate buffer, $\mathrm{pH} 7.4$, containing $0.05 \% \mathrm{CaCl}_{2}$ for 5 hours at room temperature and subsequently for 5 hours at $4^{\circ} \mathrm{C}$. Forty-micrometer sections were made with a Komatsu Electronics MB201 freezing microtome (Komatsu Electronics Inc., Tokyo, Japan). The sections were postfixed in $1 \%$ osmium tetroxide in $0.1 \mathrm{M}$ sodium cacodylate buffer, $\mathrm{pH} 7.4$, containing $0.8 \%$ potassium ferrocyanide for 60 minutes at $4^{\circ} \mathrm{C}$. Samples were then dehydrated in graded ethanol series and were subsequently embedded in Epok 812 (Okenshoji Co., Tokyo, Japan). Ultrathin sections were stained with uranyl acetate and lead citrate and were examined in a Hitachi H-7000 electron microscope (Hitachi Science Systems, Hitachinaka, Japan). To examine intercellular lipid lamellae, the protocol was modified (36): samples were postfixed in $0.2 \%$ ruthenium tetroxide (Polysciences Inc., Warrington, Pennsylvania, USA) in $0.1 \mathrm{M}$ cacodylate buffer containing $0.5 \%$ potassium ferrocyanide for 30 minutes at room temperature. To visualize the CLE, skin samples were immersed first in absolute pyridine for 2 hours prior to aldehyde fixation as described previously (37).

\section{Results}

Defective skin barrier development in TGase 1-/- mice. We have shown that $T$ Gase $1^{-/-}$neonates have impaired skin barrier function including a marked increase in TEWL (20). We first examined the development and patterning of the skin barrier in TGase $1^{-/-}$mice using an in situ skin per- meability assay. In wild-type mice at $15.5 \mathrm{dpc}$, the entire skin stained with toluidine blue (Figure 1a). At $16.5 \mathrm{dpc}$, the ventral side of wild-type embryos remained blue, but their dorsal regions were less stained, and dorsal staining varied slightly among pups at that age. In embryos after $17.5 \mathrm{dpc}$ and in neonates, the skin was wholly unstained. The staining patterns of TGase $1^{+/-}$embryos were essentially identical to that of wild-type mice at all ages (not shown). In contrast, TGase $1^{-/-}$embryos from 16.5 to 18.5 $\mathrm{dpc}$, and even TGase $1^{-/-}$neonates, were completely stained blue and no patterned staining was noted at any developmental stage. In parallel with that assay, we examined patterns of loricrin and involucrin expression in TGase $1^{-/-}$skin by immunofluorescence, but no differences in the expression of those CE precursor proteins were observed between control and TGase $1^{-/-}$mice.

The development of the skin barrier in the late gestational period was also assessed by measuring TEWL (Figure 1b). In wild type-mice, embryos at $15.5 \mathrm{dpc}$ showed markedly high levels of TEWL, as compared with neonates. At $16.5 \mathrm{dpc}$, the TEWL levels decreased slightly but significantly. After $17.5 \mathrm{dpc}$, TEWL had decreased to the level of neonates. The TEWL of TGase $1^{+/-}$mice was almost identical to that of wildtype mice at each developmental stage. In contrast, in TGase $1^{-/-}$mice, no significant decrease in TEWL was observed at $16.5 \mathrm{dpc}$, or even at 17.5 or $18.5 \mathrm{dpc}$. Those TEWL levels were similar to those of 15.5 -dpc wild-type embryos and of TGase $1^{-/-}$neonates, and thus the embryonic development of the skin barrier was defective in TGase $1^{-/-}$mice.

To assess alterations in components of the CE due to the TGase 1 deficiency, we tried to isolate remnants of insoluble CEs from 18.5-dpc TGase $1^{-/-}$epidermis. We were able to isolate intact CEs from TGase $1^{+/-}$and from wild-type epidermis, but no insoluble materials for biochemical analysis were obtained from the TGase $1^{-/}$epidermis (Figure 1c). 
Control
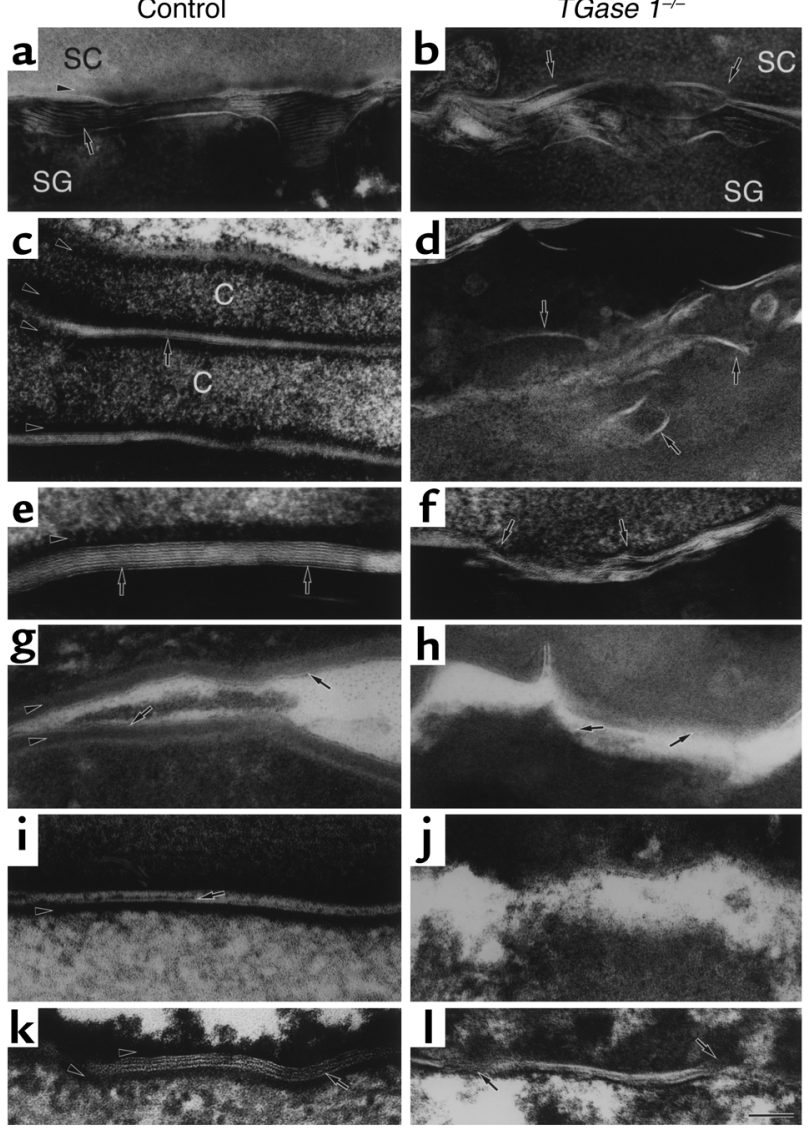

\section{Figure 2}

Abnormalities in the SC lipid lamellae and CLE of 18.5-dpc TGase $1^{-1-}$ mice. (a-f) The ultrastructure of ruthenium tetroxide-postfixed control (a, c, and $\mathbf{e})$ and TGase $1^{-/-}(\mathbf{b}, \mathbf{d}$, and $\mathbf{f})$ SC from the skin. (b) The ordered distribution of lipid membranes at the SC/SG interface is impaired. Some lipid membranes are embedded in the cells (arrows). (d) The boundaries of cornified cells are folded and the lipid lamellae are irregularly embedded in the cells (arrows). (f) The typical basic unit structure of the intercellular lamellae is not readily discernible and lamellar stacks are foreshortened (arrows). Some intercellular lamellar domains break into pieces in the cytoplasm of the cells. ( $\mathbf{g}$ and $\mathbf{h}$ ) The CLE of control (g) and TGase $1^{-/-}$mouse skin (h) visualized by pyridine treatment. (h) The CLE is absent in the TGase $1^{-/-}$mouse SC (arrows). (i-I) The ultrastructure of ruthenium tetroxide-postfixed control (i and $\mathbf{k}$ ) and TGase $1^{-/-}(\mathbf{j}$ and $\mathbf{I})$ SC from the tongue (i and $\mathbf{j}$ ) and from the forestomach (k and $\mathbf{I})$. (j) Both the CLE and the CE are lost in TGase $1^{-/-}$mice. (I) The CE is absent and the lipid lamellae are disrupted. Bar: $90 \mathrm{~nm}$ in $\mathbf{a}, \mathbf{b}$, and $\mathbf{i}-\mathbf{I} ; 100 \mathrm{~nm}$ in $\mathbf{c}$ and $\mathbf{d} ; 50 \mathrm{~nm}$ in $\mathbf{e}$; $60 \mathrm{~nm}$ in $\mathbf{f} ; 80 \mathrm{~nm}$ in $\mathbf{g}$ and $\mathbf{h}$. Arrows in $\mathbf{a}-\mathbf{f}, \mathbf{k}$, and $\mathbf{l}$, lipid membranes; arrows in $\mathbf{g}$ and $\mathbf{i}, \mathrm{CLE}$; arrowheads in $\mathbf{a}, \mathbf{c}, \mathbf{e}, \mathbf{g}$, i, and $\mathbf{k}, \mathrm{CE}$. SG, stratum granulosum; $\mathrm{C}$, corneocytes.

We have reported recently that TGase 1 is also expressed in large amounts in the lung, liver, and kidney and that it is concentrated at intercellular adherens junctions of simple epithelial cells (38). Therefore, we examined the ultrastructure of simple epithelial cells of those tissues, including the intercellular junctions in TGase $1^{-/-}$mice. In addition, we also examined intestinal epithelia and endothelial cells of the aorta. However, we found no morphological abnormalities in any of those tissues.
Defects in the SC lipid lamellae and CLE of 18.5-dpc TGase $1^{-/-}$mice. The developmental defects in the skin barrier of TGase $1^{-/-}$mice might be accompanied by disturbances in the intercellular lipid lamellae, which compose a major water permeability barrier of the SC. We visualized the ultrastructure of lipid lamellae in 18.5dpc TGase $1^{-/-}$mouse skin postfixed with ruthenium tetroxide. For these studies, we used TGase $1^{+/-}$mice as controls since no phenotypic differences were observed between wild-type and TGase $1^{+/}$mice (20). In the interface between the stratum granulosum and the SC of control epidermis, lipid membranes were released into the intercellular spaces from lamellar granules and formed regularly aligned lamellar membranes (Figure 2a). However, in TGase $1^{-/-}$mouse epidermis, the interface was irregular, and released lipid membranes failed to align properly in the intercellular spaces (Figure $2 \mathrm{~b}$ ). In the SC of control mice, corneocytes were flattened and piled up evenly (Figure 2c). The lipid lamellar membranes were arranged regularly with a repeat pattern of lucent and dense bands between adjacent CEs (Figure 2e). In contrast, the formation of the lipid lamellar structure was markedly damaged in the TGase $1^{-/-}$ mouse epidermis, where incomplete and irregularly deformed lamellae were distributed sparsely between unevenly folded cornified cells (Figure 2, d and f).

Moreover, we visualized the innermost lipid membrane, corresponding to the CLE, by pyridine treatment of the skin. In control mice, the CLE was clearly visible along the CE (Figure 2g). In contrast, the CLE as well as the $\mathrm{CE}$ was almost completely lost in the SC of TGase $1^{-/-}$mice (Figure $2 \mathrm{~h}$ ).

The lipid lamellar structures in stratified squamous epithelia of the tongue, esophagus, and forestomach were also examined. The SC was absent in the esophageal epithelia of 18.5-dpc mice in both wild-type and TGase $1^{-/-}$groups (data not shown). This is to be expected since it takes 1 month for keratinization of the mouse esophagus after birth (39). In the lingual epithelia of control mice, the lipid lamellae were not fully developed, but possible CLEs were evident in parallel with CEs (Figure 2i). In TGase $1^{-/-}$mice, the CLE appeared to be absent and the intercellular spaces were widened with irregular borders of corneocytes (Figure $2 \mathrm{j})$. In contrast to the tongue, the lipid lamellae were well developed in the forestomach epithelium from control mice (Figure 2k). However, the forestomach of TGase $1^{-/-}$mice showed irregularly arrayed lipid lamellae with interruptions (Figure 21).

Ichthyosiform phenotype of transplanted TGase $1^{-/-}$mouse skin. The gross skin morphology of TGase $1^{-/-}$neonates resembles the collodion baby phenotype with shiny, erythrodermic skin, rather than a typical case of LI with large, brownish, platelike desquamations (20). Since those neonates die soon after birth, it was impossible to follow the phenotypic changes in the skin of TGase $1^{-/-}$mice after birth. Therefore, we grafted their skin onto nude mice and analyzed its morphological alterations in the skin after birth. TGase $1^{+/-}$skin was 
also grafted onto nude mice as control. Two weeks after transplantation, the control grafts were covered with hair, as occurs in normal mouse skin (Figure 3a, left). However, skins grafted from TGase $1^{-/-}$progeny exhibited erythema with thick scales, which resembled severe ichthyosis (Figure 3a, right). The histology of control skin grafts on nude mice was similar to that of normal adult mouse skin (Figure $3 b$ ). In contrast, skins grafted from $T$ Gase $1^{-/-}$neonates showed epidermal acanthosis, severe hyperkeratosis, and immature hair follicles (Figure 3d), although TGase $1^{-/-}$neonates showed no acanthosis of the epidermis (20). Loss of epidermal pegs and rete ridges were also evident. Immunohistochemistry revealed that PCNA-positive cells were increased in the acanthotic epidermis of the grafted TGase $1^{-/-}$mouse skin (Figure $3 \mathrm{e}$ ), suggesting hyperproliferation of the epidermis.

At the ultrastructural level, skins grafted from control mice had ridged cornified layers with electrondense cytoplasms and intact CEs (Figure 4, a and b). The SC of skins grafted from TGase $1^{-/-}$mice was more compact than in 18.5-dpc TGase $1^{-/-}$mouse skin but had markedly piled up in over 80 layers (Figure 4c). The CE was still lost, and abnormal electron-dense granules were evident close to the plasma membrane (Figure 4d). These features are similar to those seen in 18.5 -dpc TGase $1^{-/-}$mice (20). In addition, numerous vacuoles were prominent in the SC of the TGase $1^{-/-}$grafted skin (Figure 4, $\mathrm{c}$ and d), and some were fused with remnants of lamellar granules (Figure 4e). Similar vacuoles were observed in the granular layers (data not shown).

Abnormalities in the intercellular lipid lamellae and in the $C L E$ s are retained in the mature TGase $1^{-/-}$mouse skin. The defective intercellular lipid lamellae and CLEs seen in 18.5-dpc TGase $1^{-/-}$mice might be repaired or altered during the maturation of the skin. To ascertain possible phenotypic changes in those structures, we next examined the ultrastructure of ruthenium tetroxide-postfixed or pyridine-treated samples from the grafted TGase $1^{-/-}$mouse skin. In skins grafted from control mice, release of lipid membranes from lamellar granules and formation of intercellular lipid lamellae at the interface of the granular and cornified layers were intact (Figure 5a). The structures of the lipid lamellae between the cornified layers were almost normal (Figure 5c). In contrast, in the grafted TGase $1^{-/-}$ mouse skins, formation of the lipid lamellae stacks was poor and retention of lamellar granules was evident (Figure 5b). In the extracellular spaces of the SC, formation of the lipid lamellae was apparently incomplete (Figure 5d). Some vacuoles surrounded by remnants of lamellar granules were noted within the cornified cells (Figure 5e). In the SC of the grafted control skin, the CLE was normally formed along the CE (Figure 5f). However, in the grafted TGase $1^{-/-}$mouse skin, the CLE as well as the CE was still completely absent (Figure $5 \mathrm{~g}$ ). Recovery of TEWL in the transplanted, ichthyosiform TGase $1^{-/-}$mouse skin. We next measured TEWL from the mature TGase $1^{-/-}$mouse skins grafted on nude mice. In grafted control skins, TEWL was $1.89 \pm 0.33 \mathrm{~g} / \mathrm{m}^{2} / \mathrm{h}$, a level similar to that found in normal neonatal skin (Figure $1 \mathrm{~b})$. The TEWL levels of TGase $1^{-/-}$neonates were very high (Figure 1b), whereas TEWL from the grafted mature TGase $1^{-/-}$mouse skin, which had massive scales, was markedly decreased to $2.41 \pm 0.46 \mathrm{~g} / \mathrm{m}^{2} / \mathrm{h}$, a level comparable to that of the grafted control skin. However, when the scales were carefully removed with forceps, TEWL was increased and was restored to $27.1 \pm 2.05$ $\mathrm{g} / \mathrm{m}^{2} / \mathrm{h}$, a level as high as the TEWL of the TGase $1^{-/-}$ neonates (Figure 1b). Thus, the thick scales generated during maturation of the TGase $1^{-/-}$skin serve to prevent water loss from the skin surface.

\section{Discussion}

In this study, we demonstrate that TGase 1 is essential to the development of the cutaneous permeability barrier. In normal development of mice, the skin barrier rapidly appears at $16 \mathrm{dpc}$ in a patterned fashion (33). Analyses of in situ dye permeability and of TEWL in embryonic mouse skin clearly reveal that the development of
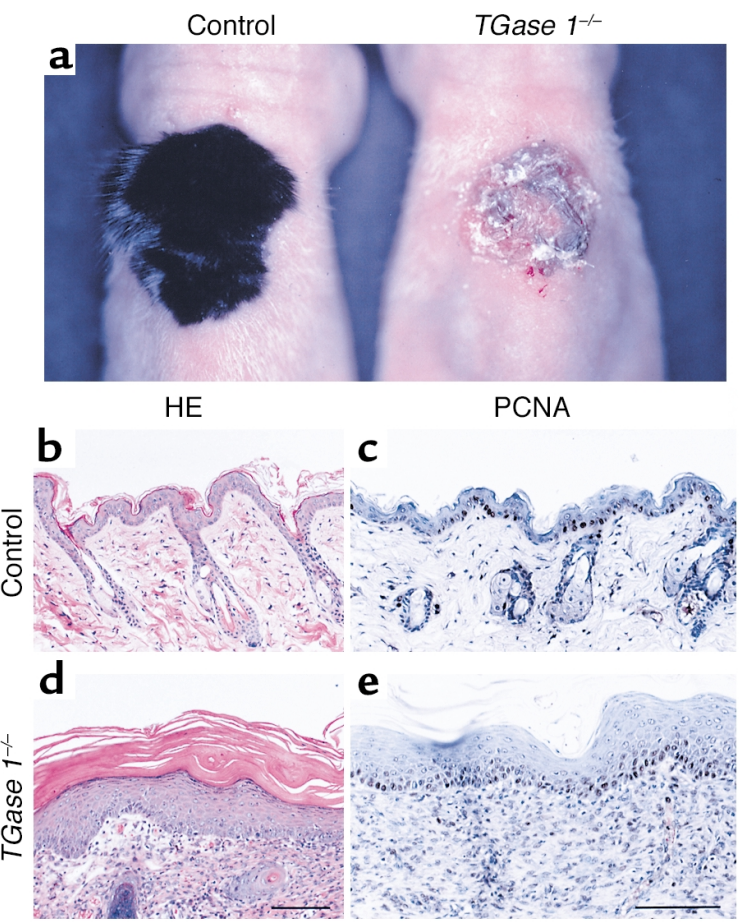

Figure 3

Ichthyosiform phenotype of mature TGase $1^{-/-}$mouse skin. (a) Gross morphology of control and TGase $1^{-1-}$ mouse skin grafted on nude mice for 2 weeks. The control graft is covered with hairs as occurs in normal mouse development, whereas the grafted TGase $1^{-/-}$mouse skin is covered with massive scales, resembling LI. (b and d) Hematoxylin-and-eosin staining (HE). The control skin graft is almost identical to normal adult mouse skin. Note the thin epidermis and the regularly arranged hair follicles $(\mathbf{b})$. In contrast, the grafted TGase $1^{-1-}$ mouse skin shows epidermal acanthosis and severe hyperkeratosis with abortive hair follicles (d). (c and e) Immunohistochemistry of PCNA. The number of PCNA-positive cells was increased in the TGase $1^{-/-}$epidermis (e), as compared with the control grafted skin (c). Bar: $140 \mu \mathrm{m}$ in $\mathbf{b}$ and $\mathbf{d}$; $100 \mu \mathrm{m}$ in $\mathbf{c}$ and $\mathbf{e}$. 
the skin barrier and its patterning is totally defective in TGase $1^{-/-}$mice. This result indicates that the impaired skin barrier function of TGase $1^{-/-}$neonates is due to defective development of the skin barrier at an embryonic stage. The patterning in the permeability barrier during development needs prior expression of loricrin, the major component of the CE (33). However, no delay or decrease in loricrin expression was evident in developing TGase $1^{-/-}$mouse skin. Instead, TGase $1^{-/-}$embryos at $18.5 \mathrm{dpc}$ show abnormalities in intercellular lipid lamellar structures of the SC, which are critical for the permeability barrier homeostasis (22). Defects in the formation of lipid lamellae cause dysregulation of the skin permeability barrier in developing TGase $1^{-/-}$mice.

The permeability barrier of the skin is regulated by organization of intercellular lipid lamellae, which depends on the formation of the CLE (31). In spite of the important role of those structures, the lipid lamellae are disorganized and the CLE as well as the CE is lost in TGase $1^{-/-}$mice. A major component of the CLE, $\omega$-hydroxyceramide, is covalently bound to protein components of the $\mathrm{CE}$ at its surface, possibly via the action of TGase 1 (30). Therefore, the loss of the CLE in TGase $1^{-/-}$mice may be caused by the dysfunction of the TGase 1 catalytic reaction required for the formation of the CLE and for the cross-linking CE, which results in disorganization of the intercellular lipid lamellae in the SC. We speculate that those incomplete structures at the cell periphery and interstices of the SC result in a loss of the rigid cellular contour and in uneven folding of the cell boundaries.

The arrangement of lipid membranes in the interstices of the SC starts at the interface of the cornified layer and the uppermost granular layer. In 18.5-dpc TGase $1^{-/-}$mice, lamellar granules containing lipid discs are produced and the contents are released into the interface almost normally. The uncoiling of the lipid discs seems to be correct there, but the alignment step of the lipid membranes appears to be uncontrolled and eventually results in the disorientation of lipid membranes and the disorganized array of lipid lamellae in the SC. Thus, the "slipping" of the lipid membranes in this step may be one characteristic of TGase $1^{-/-}$mice. This slippery outer surface of the cells in the uppermost granular layer might be due to the absence of the CLE, which plays an important role in the formation of the mature lipid lamellae.

We had initially expected that TGase 1 knockout mice would reproduce the severe ichthyosis seen in LI as they aged. Unexpectedly, however, the knockout mice died within the early neonatal period before the appearance of an ichthyosiform phenotype (20). We wondered whether not only the TGase 1 deficiency but also the postnatal environment might be involved in the pathogenesis of the ichthyosiform phenotype. Indeed, TGase $1^{-/-}$mouse skin that matures after transplantation to nude mice reveals an ichthyosiform appearance with thickening epidermis, severe hyperkeratosis, and alopecia. We have recently report- ed a substantial delay in wound healing in the mature grafted TGase $1^{-/-}$skin (35). In that report, however, we did not characterize the dramatic remodeling of the phenotype that occurs after birth and no evidence was reported about its biological or clinical meaning. In the present study, we determined that the CEs are still lost and that abnormalities in the lipid lamellae and the CLEs remain even in maturing transplanted TGase $1^{-/-}$mouse skin. Although the expression of TGase 2, TGase 3, and TGase 5 has also been found in the epidermis (40-42), those other TGase isoforms were never able to compensate for the structural abnormalities during the maturation of TGase $1^{-/-}$ skin. On the other hand, it is likely that the role of TGase 2 in development of the skin may be sufficiently compensated by other TGase isoforms, because TGase 2 knockout mice show no morphological alterations of the skin $(43,44)$.

Disorganization in the lipid lamellae has been reported in some cases of LI (32), although it is unknown whether those cases have TGase 1 mutations. In comparison with the classified ultrastructural features of ARCI, abnormalities in the CE of IC type II may be reproduced in the TGase $1^{-/-}$mouse SC as the most severe case $(15,16)$. The electron-dense aggregates that correspond to abnormal loricrin granules are evident in the grafted skin as well as in TGase $1^{-/-}$
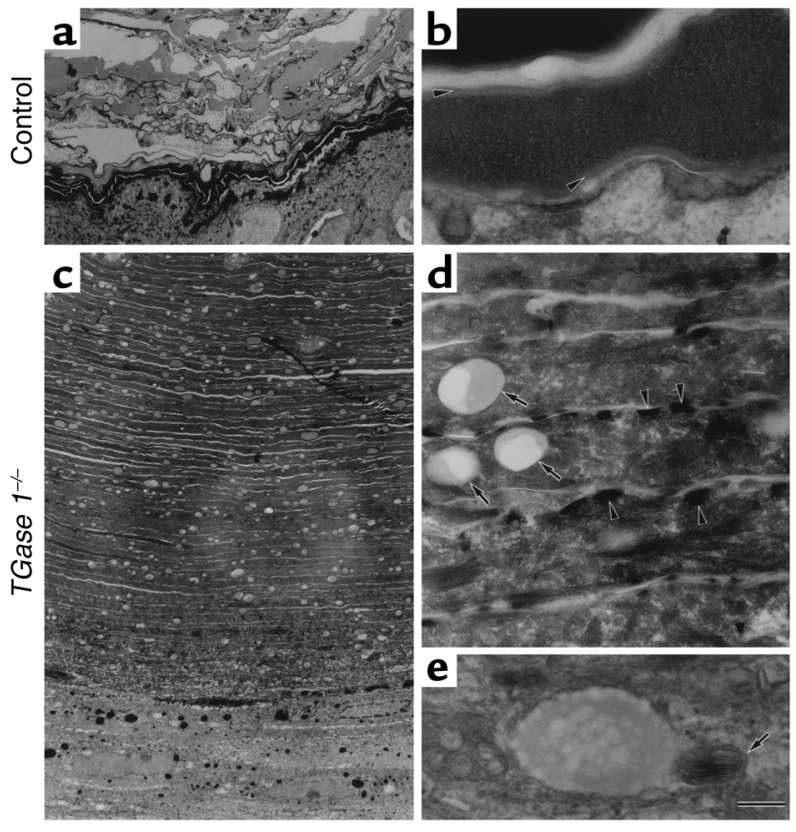

Figure 4

Ultrastructure of the grafted skin from control ( $\mathbf{a}$ and $\mathbf{b}$ ) and TGase $1^{-/-}(\mathbf{c}-\mathbf{e})$ neonates. ( $\mathbf{a}$ and $\left.\mathbf{b}\right)$ The SC of the grafted control skin. Note the electron-dense cytoplasm and the intact CE (arrowheads in b). (c) The SC of the grafted skin from TGase $1^{-/-}$mice. Note the markedly piled-up cornified cells in over 80 layers and the numerous vacuoles within the cells. (d) The CE is lost, and abnormal electron-dense granules (arrowheads) and vacuoles (arrows) are evident. (e) A vacuole fused with a remnant of a lamellar granule (arrow). Bar: $4 \mu \mathrm{m}$ in $\mathbf{a}$ and $\mathbf{c} ; 170 \mathrm{~nm}$ in $\mathbf{b}$ and $\mathbf{e} ; 440 \mathrm{~nm}$ in $\mathbf{d}$. 
Control
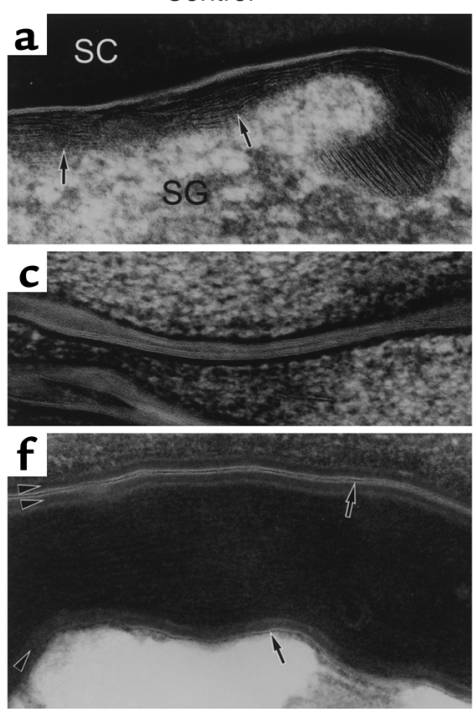

TGase 1-/

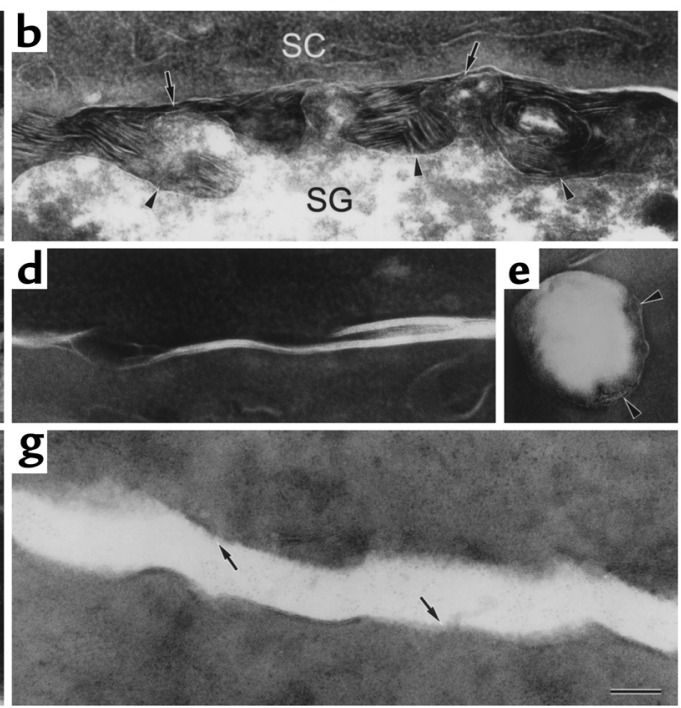

\section{Figure 5}

Sustained abnormalities of intercellular lipid lamellae and CLE in the SC of mature TGase $1^{-/-}$mouse epidermis. (a-e) Ultrastructure of ruthenium tetroxide-postfixed SC of grafted control (a and $\mathbf{c})$ and TGase $1^{-/-}(\mathbf{b}, \mathbf{d}$, and $\mathbf{e})$ mice. (a) The lipid membranes released from a lamellar granule are gliding in the SC and stratum granulosum (SG) interface of the grafted control skin (arrows). (b) Note the poor formation of lipid lamellar stacks (arrows) and the retention of lamellar granules (arrowheads). (c) The lipid lamellae are normally arranged in the grafted control skin. (d) The lipid lamellae are foreshortened and their stacking is incomplete. (e) A vacuole surrounded by remnants of lamellar granules (arrowheads). (f and $\mathbf{g}$ ) The CLE of grafted control (f) and TGase $1^{-/-}$(g) skin visualized with pyridine treatment. The CLE (arrows) is clearly visualized along the CE (arrowheads) in the grafted control skin (f). On the other hand, the CLE as well as the CE is completely absent in the grafted TGase $1^{-/-}$skin (g, arrows). Bar: $80 \mathrm{~nm}$ in a, $\mathbf{c}$, and $\mathbf{d} ; 130 \mathrm{~nm}$ in b, e, f, and $\mathbf{g}$.

neonates (20). In keeping with our finding, the characteristic abnormal granules have also been recently documented in an adult LI patient with a TGase 1 mutation (45). Vacuoles or lipid droplets reported in IC type I and in some cases of IC type II seem to correspond to vacuoles seen in the cornified cells of the mature TGase $1^{-/-}$mouse skin (14-16). The origin of those vacuoles is unknown, but some are fused with remnants of lamellar granules in the SC, suggesting that they are related to lipid metabolism. If the CLE is truly derived from the limiting membranes of lamellar granules (46), the vacuoles might be remnants of the granules composed of membranes unused for CLE formation in the TGase $1^{-/-}$mouse epidermis.

TEWL from the mature transplanted TGase $1^{-/-}$ mouse skin is, unexpectedly, as low as the control. However, when the extremely thickened scales are removed, TEWL returns to a level as high as that measured in TGase $1^{-/-}$embryos and neonates. These results suggest that the massive hyperkeratosis that develops suppresses the uncontrolled TEWL from the surface of TGase $1^{-/-}$mouse skin. In general, thickening of the $\mathrm{SC}$ and hyperplasia of the epidermis can be evoked in a low-humidity or dry environment $(47,48)$. Therefore, it is likely that exposure of TGase $1^{-/-}$mouse skin to an environment drier than that found in the uterus stimulates hyperplasia of the epidermis to produce massive scales. This regulation in the epidermis might manifest as the ichthyosiform phenotype in the mature TGase $1^{-/-}$mouse skin.
The defective barrier structures of the SC are found not only in the epidermis of TGase $1^{-/-}$mice, but also in the stratified squamous epithelia of their tongue and forestomach. In addition to dehydration, another reason for the early neonatal death of TGase $1^{-/-}$ mice might be barrier defects in those digestive organs that could affect the feeding of neonatal mice, but this is probably not a major problem because the general condition of TGase $1^{-/-}$mice is so serious that they cannot even move freely to feed. TGase 1 is also expressed in the lung, liver, and kidney and is concentrated in the intercellular adherens junction of simple epithelial cells (38). We examined TGase $1^{-/-}$mice but found no ultrastructural abnormalities in simple epithelial cells of those organs or in the endothelial cells, or in their intercellular junctions. In those tissues, other TGases might compensate for the loss of function of TGase 1, but further study will be required to explore the precise roles of TGase 1 in those cells.

In conclusion, TGase 1 is indispensable for the assembly and organization of SC barrier structures and thereby for the postnatal survival of animals. TGase 1 deficiency with abortive barrier development of the skin causes permeability defects. In maturing transplanted TGase $1^{-/-}$skin, these permeability defects can be compensated for by severe hyperkeratosis, which produces a physical barrier in order to adapt to the terrestrial environment. This adaptation process may result in the ichthyosiform skin phenotype seen in LI. 


\section{Acknowledgments}

We thank Shouji Hayashi (Cosmetics Laboratory, Kanebo Ltd.) for support and encouragement. This study was supported in part by Grants-in-Aid for Scientific Research from the Ministry of Education, Culture, Sports, Science and Technology of Japan, and by project grants of the Center for Molecular Medicine of Jichi Medical School from the Ministry of Education, Culture, Sports, Science, and Technology of Japan.

1. Vandersteen, P., Muller, S., and Minn, R. 1972. Lamellar ichthyosis: an enzyme histochemical, light, and electron microscopic study. Arch. Dermatol. 106:694-701.

2. Williams, M.L., and Elias, P.M. 1985. Heterogeneity in autosomal recessive ichthyosis: clinical and biochemical differentiation of lamellar ichthyosis and nonbullous congenital ichthyosiform erythroderma. Arch. Dermatol. 121:477-488.

3. Hazell, M., and Marks, R. 1985. Clinical, histologic, and cell kinetic discriminants between lamellar ichthyosis and nonbullous congenital ichthyosiform erythroderma. Arch. Dermatol. 121:489-493.

4. Frenck, E., and Mevorah, B. 1977. The keratinization disorder in collodion babies evolving into lamellar ichthyosis. J. Cutan. Pathol. 4:329-337.

5. Buyse, L., Graves, C., Wijeyesekera, K., Alfaham, M., and Finlay, A. 1993. Collodion baby dehydration: the danger of high transepidermal water loss. Br. J. Dermatol. 129:86-88.

6. Laiho, E., et al. 1997. Transglutaminase 1 mutations in autosomal recessive congenital ichthyosis: private and recurrent mutations in an isolated population. Am. J. Hum. Genet. 61:529-538.

7. Russell, L.J., et al. 1995. Mutations in the gene for transglutaminase 1 in autosomal recessive lamellar ichthyosis. Nat. Genet. 9:279-283.

8. Huber, M., et al. 1995. Mutations of keratinocyte transglutaminase in lamellar ichthyosis. Science. 267:525-528.

9. Parmentier, L., et al. 1996. Mapping of a second locus for lamellar ichthyosis to chromosome 2q33-35. Hum. Mol. Genet. 5:555-559.

10. Fischer, J., et al. 2000. Two new loci for autosomal recessive ichthyosis on chromosomes $3 \mathrm{p} 21$ and $19 \mathrm{p} 12$-q 12 and evidence for further genetic heterogeneity. Am. J. Hum. Genet. 66:904-913.

11. Hennies, H.C., Küster, W., Wiebe, V., Krebsová, A., and Reis, A. 1998. Genotype/phenotype correlation in autosomal recessive lamellar ichthyosis. Am. J. Hum. Genet. 62:1052-1061.

12. Virolainen, E., et al. 2000. Assignment of a novel locus for autosomal recessive congenital ichthyosis chromosome 19p13.1-p13.2. Am. J. Hum. Genet. 66:1132-1137.

13. Anton-Lamprecht, I. 1992. The skin. In Diagnostic ultrastructure of non-neoplastic diseases. J.M. Papadimitriou, D.W. Henderson, and D.V. Spagnolo, editors. Churchill Livingstone. Edinburgh, United Kingdom. 460-468.

14. Niemi, K.-M., Kanerva, L., Kuokkanen, K., and Ignatius, J. 1994. Clinical, light and electron microscopic features of recessive congenital ichthyosis type I. Br. J. Dermatol. 130:626-633.

15. Niemi, K.-M., Kanerva, L., and Kuokkanen, K. 1991. Recessive ichthyosis congenita type II. Arch. Dermatol. Res. 283:211-218.

16. Laiho, E., et al. 1999. Clinical and morphological correlations for transglutaminase 1 gene mutations in autosomal recessive congenital ichthyosis. Eur. J. Hum. Genet. 7:625-632.

17. Greenberg, C.S., Birckbichler, P.J., and Rice, R.H. 1991. Transglutaminases: multifunctional cross-linking enzymes that stabilize tissues. FASEB J. 5:3071-3077.

18. Nemes, Z., and Steinert, P.M. 1999. Bricks and mortar of the epidermal barrier. Exp. Mol. Med. 31:5-19.

19. Candi, E., et al. 1995. Biochemical, structural, and transglutaminase substrate properties of human loricrin, the major epidermal cornified cell envelope protein. J. Biol. Chem. 270:26382-26390.

20. Matsuki, M., et al. 1998. Defective stratum corneum and early neonatal death in mice lacking the gene for transglutaminase 1 (keratinocyte transglutaminase). Proc. Natl. Acad. Sci. USA. 95:1044-1049.

21. Huber, M., et al. 1997. Consequences of seven novel mutations on the expression and structure of keratinocyte transglutaminase. J. Biol. Chem. 272:21018-21026.

22. Wertz, P.W. 2000. Lipids and barrier function of the skin. Acta Derm. Venereol. Suppl. (Stockh.) 208:7-11.
23. Elias, P.M., and Menon, G.K. 1991. Structural and lipid biochemical correlates of the epidermal permeability barrier. Adv. Lipid Res. 24:1-26.

24. Landmann, L. 1986. Epidermal permeability barrier: transformation of lamellar granule-disks into intercellular sheets by a membrane-fusion process, a freeze-fracture study. J. Invest. Dermatol. 87:202-209.

25. Grayson, S., and Elias, P.M. 1982. Isolation and lipid biochemical characterization of stratum corneum membrane complexes: implications for the cutaneous permeability barrier. J. Invest. Dermatol. 78:128-135.

26. Swartzendruber, D.C., Wertz, P.W., Madison, K.C., and Downing, D.T. 1989. Evidence that the corneocyte has a chemically bound lipid envelope. J. Invest. Dermatol. 88:709-713.

27. Swartzendruber, D.C., Kitko, D.J., Wertz, P.W., Madison, K.C., and Downing, D.T. 1988. Isolation of corneocyte envelopes from porcine epidermis. Arch. Dermatol. Res. 280:424-429.

28. Wertz, P.W., Madison, K.C., and Downing, D.T. 1989. Covalently bound lipids of human stratum corneum. J. Invest. Dermatol. 92:109-111.

29. Marekov, L.N., and Steinert, P.M. 1998. Ceramides are bound to structural proteins of the human foreskin epidermal cornified cell envelope. J. Biol. Chem. 273:17763-17770.

30. Nemes, Z., Marekov, L.N., Fesus, L., and Steinert, P.M. 1999. A novel function for transglutaminase 1: attachment of long-chain omegahydroxyceramides to involucrin by ester bond formation. Proc. Natl. Acad. Sci. USA. 96:8402-8407.

31. Downing, D.T. 1992. Lipid and protein structures in the permeability barrier of mammalian epidermis. J. Lipid Res. 33:301-313.

32. Ghadially, R., Williams, M.L., Hou, S.Y.E., and Elias, P.M. 1992. Membrane structural abnormalities in the stratum corneum of the autosomal recessive ichthyoses. J. Invest. Dermatol. 99:755-763.

33. Hardman, M.J., Sisi, P., Banbury, D.N., and Byrne, C. 1998. Patterned acquisition of skin barrier function during development. Differentiation. 125:1541-1552.

34. Mehrel, T., et al. 1990. Identification of a major keratinocyte cell envelope protein, loricrin. Cell. 61:1103-1112.

35. Inada, R., et al. 2000. Facilitated wound healing by activation of the Transglutaminase 1 gene. Am. J. Pathol. 157:1875-1882.

36. Swartzendruber, D.C., Wertz, P.W., Kitko, D.J., Madison, K.C., and Downing, D.T. 1989. Molecular models of the intercellular lipid lamellae in mammalian stratum corneum. J. Invest. Dermatol. 92:251-257.

37. Behne, M., et al. 2000. Omega-hydroxyceramides are required for corneocyte lipid envelope (CLE) formation and normal epidermal permeability barrier function. J. Invest. Dermatol. 114:185-192.

38. Hiiragi, T., et al. 1999. Transglutaminase type 1 and its cross-linking activity are concentrated at adherens junctions in simple epithelial cells. J. Biol. Chem. 274:34148-34154.

39. Duan, H., Gao, F., Li, S., and Nagata, T. 1993. Postnatal development and aging of esophageal epithelium in mouse: a light and electron microscopic radioautographic study. Cell. Mol. Biol. (Noisy-le-grand). 39:309-316.

40. Kim, H.-C., et al. 1991. The complete amino acid sequence of the human transglutaminase K enzyme deduced from the nucleic acid sequences of cDNA clones. J. Biol. Chem. 266:536-539.

41. Aeschlimann, D., Koeller, M.K., Allen-Hoffmann, B.L., and Mosher, D.F. 1998. Isolation of a cDNA encoding a novel member of the transglutaminase gene family from human keratinocytes: detection and identification of transglutaminase gene products based on reverse transcription polymerase chain reaction with degenerate primers. J. Biol. Chem. 273:3452-3460.

42. Candi, E., et al. 2001. Transglutaminase 5 cross-links loricrin, involucrin, and small proline-rich proteins in vitro. J. Biol. Chem. 276:35014-35023.

43. De Laurenzi, V., and Melino, G. 2001. Gene disruption of tissue transglutaminase. Mol. Cell. Biol. 21:148-155.

44. Nanda, N., et al. 2001. Targeted inactivation of Gh/transglutaminase II. J. Biol. Chem. 276:20673-20678.

45. Yotsumoto, S., et al. 2000. Analyses of the transglutaminase 1 gene mutation and ultrastructural characteristics in a Japanese patient with lamellar ichthyosis. J. Dermatol. Sci. 24:119-125.

46. Elias, P.M., et al. 2000. Origin of the corneocyte lipid envelope (CLE): observations in harlequin ichthyosis and cultured human keratinocytes. J. Invest. Dermatol. 115:765-769.

47. Denda, M., et al. 1998. Exposure to a dry environment enhances epidermal permeability barrier function. J. Invest. Dermatol. 111:858-863.

48. Denda, M., Sato, J., Tsuchiya, T., Elias, P.M., and Feingold, K.R. 1998. Low humidity stimulates epidermal DNA synthesis and amplifies the hyperproliferative response to barrier disruption: implication for seasonal exacerbations of inflammatory dermatoses. J. Invest. Dermatol. 111:873-878. 